\title{
Controlling Pattern Formation in Nanoparticle Assemblies via Directed Solvent Dewetting
}

\author{
Christopher P. Martin, Matthew O. Blunt, Emmanuelle Pauliac-Vaujour, Andrew Stannard, and Philip Moriarty* \\ School of Physics and Astronomy, The University of Nottingham, University Park, Nottingham NG7 2RD, United Kingdom
}

Ioan Vancea and Uwe Thiele

Max-Planck-Institut für Physik komplexer Systeme, Nöthnitzer Strasse 38, D-01187 Dresden, Germany (Received 24 May 2007; published 14 September 2007)

\begin{abstract}
We have achieved highly localized control of pattern formation in two-dimensional nanoparticle assemblies by direct modification of solvent dewetting dynamics. A striking dependence of nanoparticle organization on the size of atomic force microscope-generated surface heterogeneities is observed and reproduced in numerical simulations. Nanoscale features induce a rupture of the solvent-nanoparticle film, causing the local flow of solvent to carry nanoparticles into confinement. Microscale heterogeneities instead slow the evaporation of the solvent, producing a remarkably abrupt interface between different nanoparticle patterns.
\end{abstract}

DOI: 10.1103/PhysRevLett.99.116103

PACS numbers: 68.05.Cf, 61.46.Df, 68.08.Bc

Colloidal solutions of nanoparticles are simple to synthesize, exceptionally stable, and can be made from materials with a wide variety of chemical and electrical properties. Their versatility makes them ideal building blocks for the next generation of nanoscale electronic devices. When deposited on a surface, colloidal nanoparticles form a diverse array of patterns [1-8] for which the behavior of the solvent plays a key role. Isolated islands, wormlike domains, continuous labyrinthine patterns, and polygonal networks can each be produced by varying the experimental conditions $[1,5,9,10]$. Although there have been impressive recent examples of the exploitation of dewetting to form stripes of nanoparticles $[6,7,11]$ and the use of structured substrates to direct dewetting in polymer systems has been studied in some depth [1214], spatial control of dewetting-induced pattern formation in $2 \mathrm{D}$ assemblies of nanoparticles has to date not been demonstrated.

Here we describe highly localized control of 2D pattern formation in colloidal nanoparticle arrays via surface inhomogeneities created by atomic force microscope (AFM)-induced oxidation. In addition to representing a new technique for nanostructure control, our experiments and associated simulations provide key insights into the mechanisms of surface inhomogeneity-directed selforganization in nanoparticle systems. Whereas nanoscale surface features drive the rupture of the solventnanoparticle film, microscale oxide features on an otherwise hydrophobic surface retard solvent evaporation, providing important complementary strategies for spatial control of dewetting dynamics. We also show that a simple modification of the Hamiltonian introduced by Rabani et al. [9] can reproduce important experimentally observed patterns not captured by the original algorithm.

Our experiments are based on AFM-induced oxidation of hydrogen-passivated $\mathrm{Si}(111)$ surfaces $[15,16]$. An Asylum Research MFP-3D AFM system operating in in- termittent contact mode with closed-loop control was used for both imaging and local oxidation of the $\mathrm{H}: \mathrm{Si}(111)$ samples. Oxidation was carried out using PtIr-coated silicon probes at a bias of $-10 \mathrm{~V}$ and in $70 \%$ humidity. Octanethiol-passivated $\mathrm{Au}$ nanoparticles of $\sim 2 \mathrm{~nm}$ core diameter $( \pm 8 \%)$ were prepared in toluene [17]. For experiments involving rapid solvent evaporation, a $25 \mu \mathrm{l}$ droplet of an appropriately diluted solution (typical concentration values ranged from $0.1 \mathrm{mg} / \mathrm{ml}$ to $1 \mathrm{mg} / \mathrm{ml}$ ) was placed on the locally oxidized substrate and the sample subsequently spun at $4 \mathrm{k} \mathrm{rpm}$. Slow evaporation was induced by keeping the solvent in a meniscus on the sample by means of a small Teflon ring.

The 2D Ising model simulations described by Rabani et al. [9] are capable of replicating a large number of the nanoparticle structures seen in experiment. In particular, certain classes of cellular structure are reproduced with an accuracy that renders them statistically indistinguishable from experiment $[10,18]$. There are, however, a number of common experimental phenomena that cannot be simulated by this method and which are of key importance to our studies of directed dewetting. For example, Fig. 1(a) shows a structure that is frequently observed in our experiments. A Fourier transform [19] reveals that the pattern exhibits not just one but two preferred cell sizes, each having a relatively wide distribution. The nature of the structures produced by the simulation is governed by the parameters in the Hamiltonian

$$
E=-\epsilon_{l} \sum_{\langle i j\rangle} l_{i} l_{j}-\epsilon_{n} \sum_{\langle i j\rangle} n_{i} n_{j}-\epsilon_{n l} \sum_{\langle i j\rangle} n_{i} l_{j}-\mu \sum_{i} l_{i},
$$

where $\epsilon_{l}, \epsilon_{n}$, and $\epsilon_{n l}$ are the liquid-liquid, nanoparticlenanoparticle, and nanoparticle-liquid interaction energies, respectively. As discussed by Rabani et al. [9], the value of $\mu$ (the solvent chemical potential) determines the mean equilibrium density of solvent and is an effective parameter 


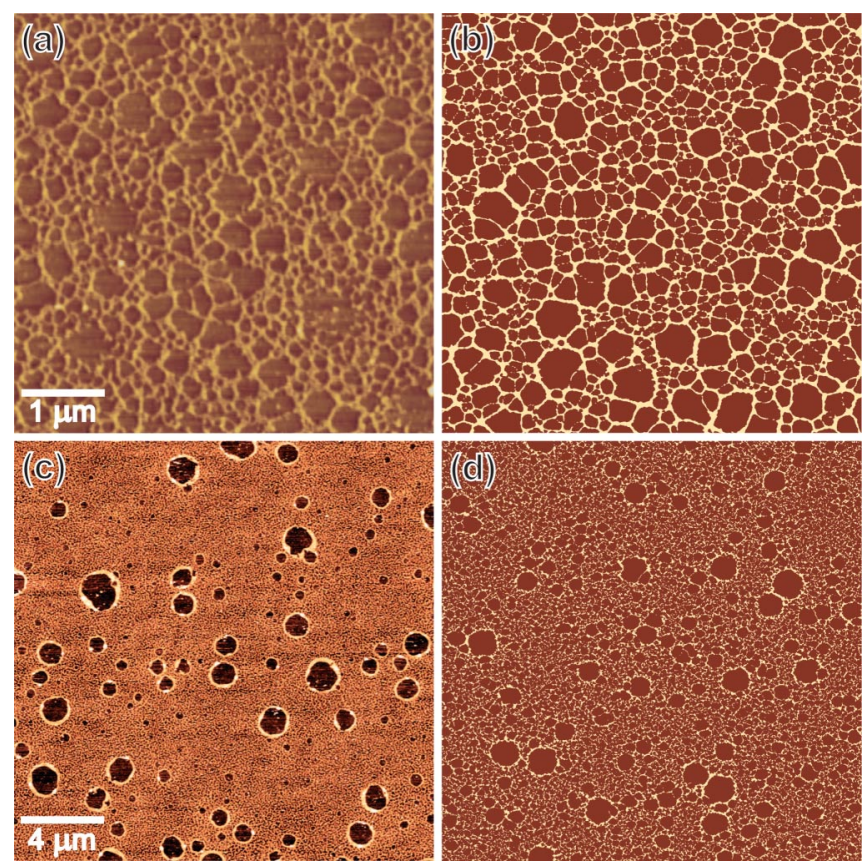

FIG. 1 (color online). (a) An AFM image of a two-level cellular structure formed by spin-casting gold nanoparticles in toluene onto a silicon substrate, (b) a simulated structure formed by linking the chemical potential $(\mu)$ to the solvent density [in the form $\mu(v)=\mu_{0}+\left(0.1 \mu_{0} \times v^{0.7}\right)$, where $v$ is the fraction of solvent that has become vapor, and $\mu_{0}$ is the value of $\mu$ at the beginning of the simulation], (c) an AFM image of gold nanoparticles spun onto silicon from chloroform, and (d) a simulated image with a much stronger coupling between $\mu$ and solvent coverage [in the form $\mu(v)=\mu_{0}+\left(0.5 \mu_{0} \times v^{0.7}\right)$ ].

representing any interaction energy that is not explicitly taken into account by the other parameters.

Although the original model was two dimensional, Sztrum et al. [20] have recently extended it to three dimensions to explain nanoparticle stalagmites. However, the pattern-forming processes we discuss here occur within the final few nanometers of the evaporating solvent $[9,21]$. Therefore, the physical nanoparticle "subsystem" is truly 2D whereas the solvent "subsystem" retains some 3D aspects. In particular, the interaction of the solvent with the substrate and therefore its evaporation rate depends on the remaining amount of solvent. We find that a simple change to the Hamiltonian of the original 2D model is sufficient to capture the resulting partial 3D nature. Specifically, by making the chemical potential an explicit function of the global solvent density, we can model an increase in $\mu$ as the solvent layer thins during evaporation. This density dependence corresponds to a thicknessdependent disjoining pressure [22] in our "pseudo-3D" approach, accounting for substrate wettability in continuum models [23,24]. As a result, the solvent evaporates with a rate inversely proportional to its coverage (Fig. 1). Figure 1(b) shows the result of this modification, which matches the experimental image not just qualitatively, but also quantitatively $[18,19]$. Increasing the coupling be- tween $\mu$ and solvent coverage [Fig. 1(d)] replicates the effect of using chloroform in the experiments [Fig. 1(c)]. Importantly, the nanoparticle rings [25-27] in these simulated structures are formed spontaneously, without the manual introduction of nucleation sites described by Yosef and Rabani [27] — a technique discussed below.

The behavior of the solvent plays a critical role in determining the nature of the nanoparticle patterns; to a large extent, the particles are merely passengers on the tide of the solvent, and their final positions describe the history of flow and evaporation. Modifying the surface of the substrate to perturb the behavior of the solvent must therefore lead to a modification of the nanoparticle structures.

Figure 2(a) shows the striking effect on nanoparticle organization of an AFM-oxidized $4 \times 4 \mu \mathrm{m}^{2}$ square on the bare substrate $[15,16]$. There is an extremely sharp transition from a cellular structure on the oxide to a broken wormlike pattern elsewhere. Given that the edge of the oxide region should in principle represent a steep gradient in wettability, with strong implications for dewettingmediated pattern formation [28,29], the lack of a region denuded of nanoparticles at the edge of the oxide is intriguing. We return to a discussion of this important point below. Simulations indicate that cellular networks are generated by slower solvent evaporation than that which occurs in the formation of labyrinthine structures $[9,18]$. This
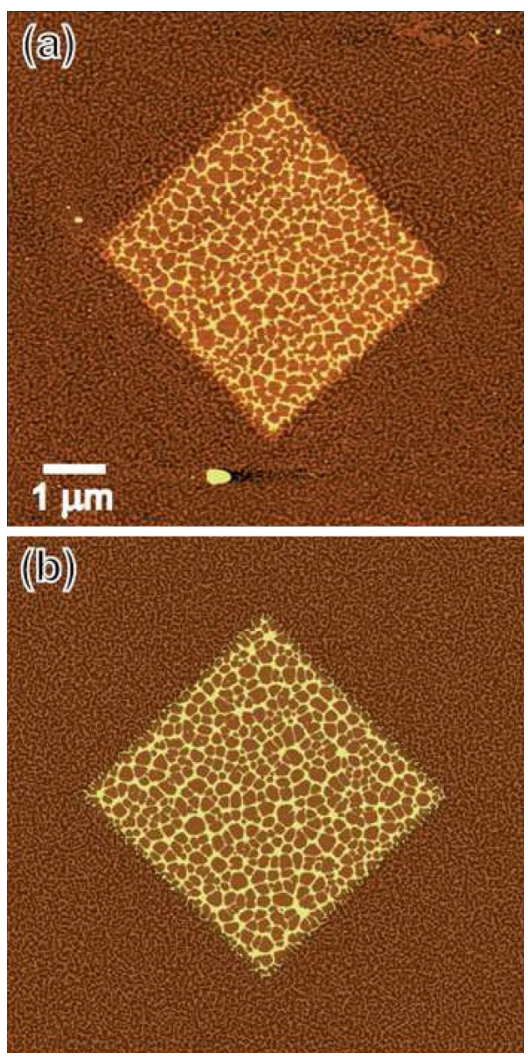

FIG. 2 (color online). (a) An AFM image of gold nanoparticles spun from toluene onto $\mathrm{H}: \mathrm{Si}(111)$ with an AFM-patterned $4 \mu \mathrm{m} \times 4 \mu \mathrm{m}$ square of oxide in the center and (b) the result of a simulation with $|\mu| 6 \%$ lower on the oxide area. 
would suggest that evaporation is somehow retarded by the presence of the oxide; i.e., the solvent finds the oxide areas more wettable. Figure 2(b) shows the result of spatially varying the value of $\mu$, such that its modulus is very slightly $(6 \%)$ lower on the oxide than in the surrounding area, with an interface region comprising a $100 \mathrm{~nm}$ linear transition. This equates to a greater wettability in the region of the oxide. The image shown in Fig. 2(b) matches the experimental result extremely well.

Contact angle measurements show, however, that there is no observable difference in the wettability of macroscopic volumes of toluene on $\mathrm{H}: \mathrm{Si}(111)$ and native oxideterminated $\mathrm{Si}(111)$ surfaces having comparable roughness. Both substrates yield a contact angle close to zero $\left(\approx 5^{\circ}\right)$, albeit with a very large error bar $( \pm 50 \%)$. These measurements would seem to be at odds not only with the results of Fig. 2, where a greater wettability of the oxide region is observed, but with estimates of Hamaker constants [22] which yield different signs for the air-toluene-silicon and air-toluene-silicon oxide systems. We can, however, explain these apparent discrepancies between wetting theory and experiment in terms of the nanoscale structure of the SiO-Si system. First, as pointed out by Seeman et al. [30], the appropriate system to consider for the estimation of Hamaker constants is air-toluene-SiO-Si where the native oxide layer is $\approx 2 \mathrm{~nm}$ thick. In this context, the high wettability of toluene on native oxide-terminated silicon seen in the contact angle measurements is not unexpected. Second, and of key importance for our directed dewetting experiments, the stronger affinity of the apolar solvent for the oxide region rather than for the bare surface in Fig. 2 can largely be rationalized in terms of the higher degree of roughness/porosity of silicon oxide formed using scanning probe nanolithography as compared to oxides prepared by more conventional methods [31,32].

Below a critical feature linewidth $(\approx 200 \mathrm{~nm})$, the effect of oxide regions on the far-from-equilibrium flow of solvent is radically different from that shown in Fig. 2. Figure 3(a) shows nanoparticle patterns formed in the presence of oxide rings of $1 \mu \mathrm{m}$ diameter. It is possible to reproduce this effect in our simulations by completely removing sections of solvent and nanoparticles from regions representing the oxide rings prior to the start of the simulation. This is similar to the technique employed by Yosef and Rabani [27].

The importance of dewetting nucleated by a gradient in wettability has been discussed in some depth for thin polymer films on heterogeneous substrates $[28,29]$. While a wettability gradient exists for the AFM-defined patterns shown in both Fig. 2 and 3, it is only for the latter that a denuded zone free of nanoparticles is observed in the vicinity of the surface heterogeneity, indicating that the feature linewidth plays a fundamental role in driving the rupture of the solvent-nanoparticle film [19]. In addition to the chemical heterogeneity represented by the oxide regions, as the solvent thins by evaporation, there comes a point when the $3 \mathrm{~nm}$ height difference of the small oxide

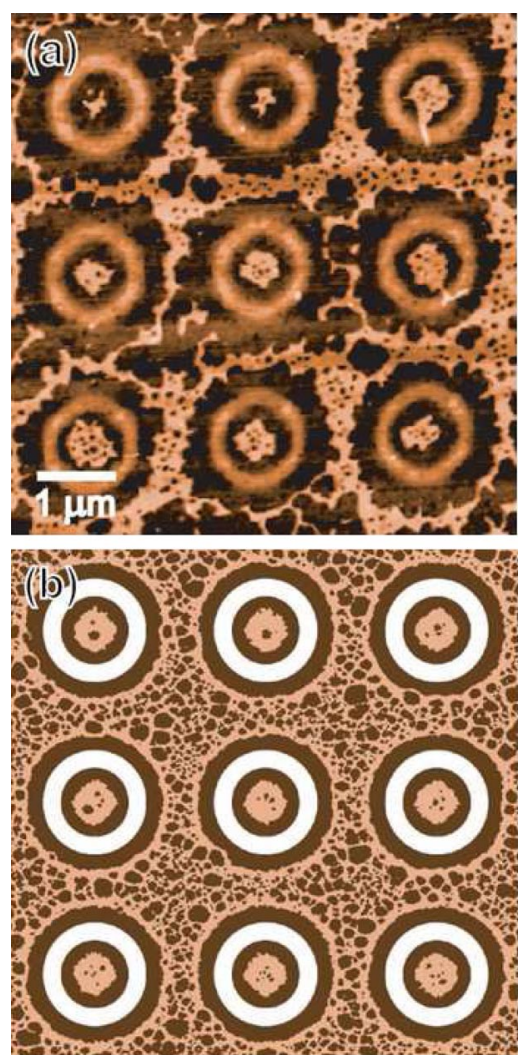

FIG. 3 (color online). (a) An AFM image showing the effect of $1 \mu \mathrm{m}$ diameter rings of oxide on nanoparticle pattern formation. The oxide features in the image are almost entirely free of $\mathrm{Au}$ nanoparticles and a denuded zone extends round each ring for a further $100 \mathrm{~nm}$. In the regions between the rings, the Au nanoparticle layer forms a network arising from nucleated dewetting of the solvent. (b) A simulation in which sections of solvent and particles have been removed (indicated in white) to approximate the effect of dewetting from the oxide regions before the realm of the simulation.

regions becomes very significant. These regions are likely to be the first in which the thickness of the film becomes small enough for the disjoining pressure to cause nucleation of a hole in the film. This tailor-made hole will then grow by evaporation, isolating a small group of nanoparticles inside the ring. A simulation including this modification, and also a solvent density-dependent chemical potential (see Fig. 1), is shown in Fig. 3(b). This directed dewetting process enables confinement within specific nanoscale surface regions without the need for chemical functionalization of the nanoparticles.

The effect of the greater roughness of the AFM-oxidized regions can be exploited in an experimental regime that is much closer to equilibrium conditions. The oxide square in Fig. 4(a) has more than twice the rms roughness of the surrounding surface. When a nanoparticle solution is allowed to evaporate from this surface over a period of hours rather than seconds, the significantly decreased mobility of the particles on the rougher areas becomes apparent. Particles that enter the oxide region are "trapped," resulting in a dense nanoparticle coverage of the square 

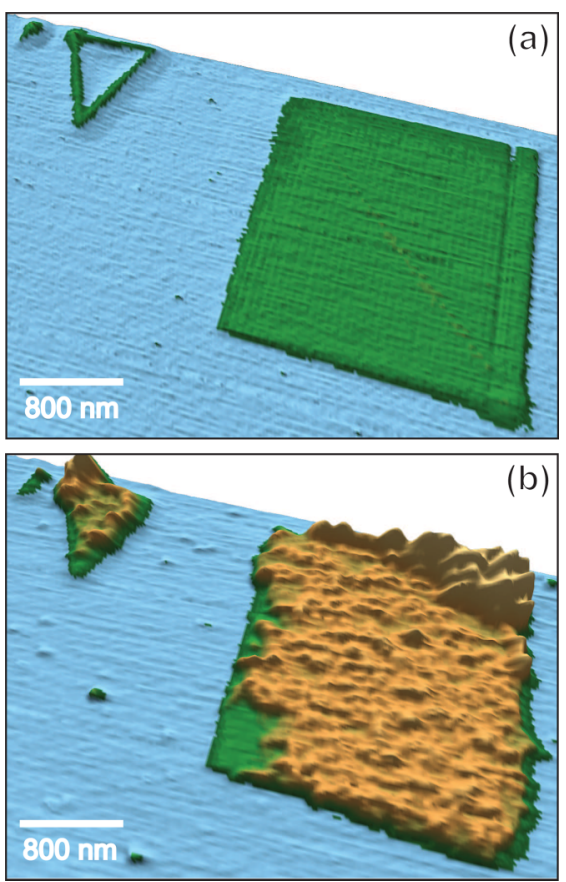

FIG. 4 (color online). (a) A three-dimensional rendering of an AFM image of raised oxide areas on $\mathrm{H}: \mathrm{Si}(111)$ and (b) the same areas covered with trapped nanoparticles after slow evaporation of a gold nanoparticle solution.

[Fig. 4(b)]. Moreover, we find that a triangular "box" with oxidized boundaries just a few tens of nm wide [upper left corner of Fig. 4(a)] has a similar trapping effect [Fig. 4(b)].

The ability to control pattern formation by locally directing solvent dewetting is an important development on the road to self-organized nanoparticle devices. A lack of requirement for particle functionalization allows this technique to be employed with a wide range of different particle types. The approach we describe thus opens up a rich new parameter space in directing the assembly of nanoparticle arrays.

We are grateful for the financial support of the UK EPSRC and the EU Framework Programme 6 Marie Curie scheme [under Grant No. MRTN-CT-2004005728 (PATTERNS)]. We would also like to thank Morgan Alexander for help with the contact angle measurements and to very gratefully acknowledge helpful discussions with James Sharp, Ulli Steiner, and Coen van den Brom

*philip.moriarty@ nottingham.ac.uk

[1] G. Ge and L. Brus, J. Phys. Chem. B 104, 9573 (2000).

[2] P. Moriarty, M.D. R. Taylor, and M. Brust, Phys. Rev. Lett. 89, 248303 (2002).

[3] S. Narayanan, J. Wang, and X.-M. Lin, Phys. Rev. Lett. 93, 135503 (2004).

[4] T.P. Bigioni, X.-M. Lin, T. T. Nguyen, E. I. Corwin, T. A. Witten, and H. M. Jaeger, Nat. Mater. 5, 265 (2006).
[5] M. O. Blunt, C. P. Martin, M. Ahola-Tuomi, E. PauliacVaujour, P. Sharp, P. Nativo, M. Brust, and P. J. Moriarty, Nature Nanotechnology 2, 167 (2007).

[6] J. Huang, F. Kim, A. R. Tao, S. Connor, and P. Yang, Nat. Mater. 4, 896 (2005).

[7] N. Lu et al., Nano Lett. 4, 885 (2004).

[8] A. J. Parker, P. A. Childs, R. E. Palmer, and M. Brust, Nanotechnology 12, 6 (2001).

[9] E. Rabani, D. R. Reichman, P. L. Geissler, and L. E. Brus, Nature (London) 426, 271 (2003).

[10] C. Martin, M. O. Blunt, E. Pauliac-Vaujour, P. Moriarty, A. Fahmi, A. D'Aleo, L. D. Cola, and F. Vogtle, Systems Self-Assembly: Interdisciplinary Snapshots, edited by N. Krasnogor, S. Gustafson, D. Pelta, and J. L. Verdegay (Elsevier, New York, 2007).

[11] J. Xu, J. Xia, and Z. Lin, Angew. Chem., Int. Ed. 46, 1860 (2007).

[12] Z. Zhang, Z. Wang, R. Xing, and Y. Han, Polymer 44, 3737 (2003).

[13] D. Julthongpiput, W. Zhang, J. F. Douglas, A. Karim, and M. J. Fasolka, Soft Matter 3, 613 (2007).

[14] M. Geoghegan and G. Krausch, Prog. Polym. Sci. 28, 261 (2003).

[15] P. Avouris, R. Martel, T. Hertel, and R. Sandstrom, Appl. Phys. A 66, S659 (1998).

[16] J. A. Dagata, Science 270, 1625 (1995).

[17] M. Brust, M. Walker, D. Bethell, D. J. Schiffrin, and R. Whyman, Journal of the Chemical Society-Chemical Communications 7, 801 (1994).

[18] C. P. Martin, M. O. Blunt, and P. Moriarty, Nano Lett. 4, 2389 (2004).

[19] See EPAPS Document No. E-PRLTAO-99-024737 for (1) a description of Fourier transform, Voronoi tesselation, and Minkowski morphometry analyses for AFM images of nanoparticle assemblies and (2) an AFM image (and associated brief discussion) of directed dewetting leading to formation of nanoparticle rings. For more information on EPAPS, see http://www.aip.org/pubservs/epaps.html.

[20] C. G. Sztrum, O. Hod, and E. Rabani, J. Phys. Chem. B 109, 6741 (2005)

[21] E. Pauliac-Vaujour and P. Moriarty (unpublished).

[22] J. N. Israelachvili, Intermolecular and Surface Forces (Academic, New York, 1992), 2nd ed.

[23] P. G. de Gennes, Rev. Mod. Phys. 57, 827 (1985).

[24] U. Thiele, Eur. Phys. J. E 12, 409 (2003).

[25] P. C. Ohara and W. M. Gelbart, Langmuir 14, 3418 (1998).

[26] M. Maillard, L. Motte, and M. P. Pileni, Adv. Mater. 13, 200 (2001).

[27] G. Yosef and E. Rabani, J. Phys. Chem. B 110, 20965 (2006).

[28] R. Konnur, K. Kargupta, and A. Sharma, Phys. Rev. Lett. 84, 931 (2000).

[29] L. Brusch, H. Kühne, U. Thiele, and M. Bär, Phys. Rev. E 66, 011602 (2002).

[30] R. Seemann, S. Herminghaus, and K. Jacobs, Phys. Rev. Lett. 86, 5534 (2001).

[31] P. Avouris, T. Hertel, and R. Martel, Appl. Phys. Lett. 71, 285 (1997).

[32] J. Bico, U. Thiele, and D. Quéré, Colloids and Surfaces A 206, 41 (2002). 